\title{
Supporting Information \\ Printed and Laser-Activated Liquid Metal-Elastomer Conductors Enabled by Ethanol/PDMS/Liquid Metal Double Emulsions
}

Shanliangzi Liu ${ }^{a, b}$, Sang Yup Kim ${ }^{a}$, Kristen E. Henry ${ }^{a}$, Dylan S. Shah, Rebecca Kramer-Bottiglio ${ }^{a *}$

${ }^{a}$ School of Engineering and Applied Science, Yale University,10 Hillhouse Avenue, New Haven, CT 06520, USA.

${ }^{b}$ School of Mechanical Engineering, Purdue University, 585 Purdue Mall, West Lafayette, IN 47907, USA.

*email: rebecca.kramer@yale.edu 

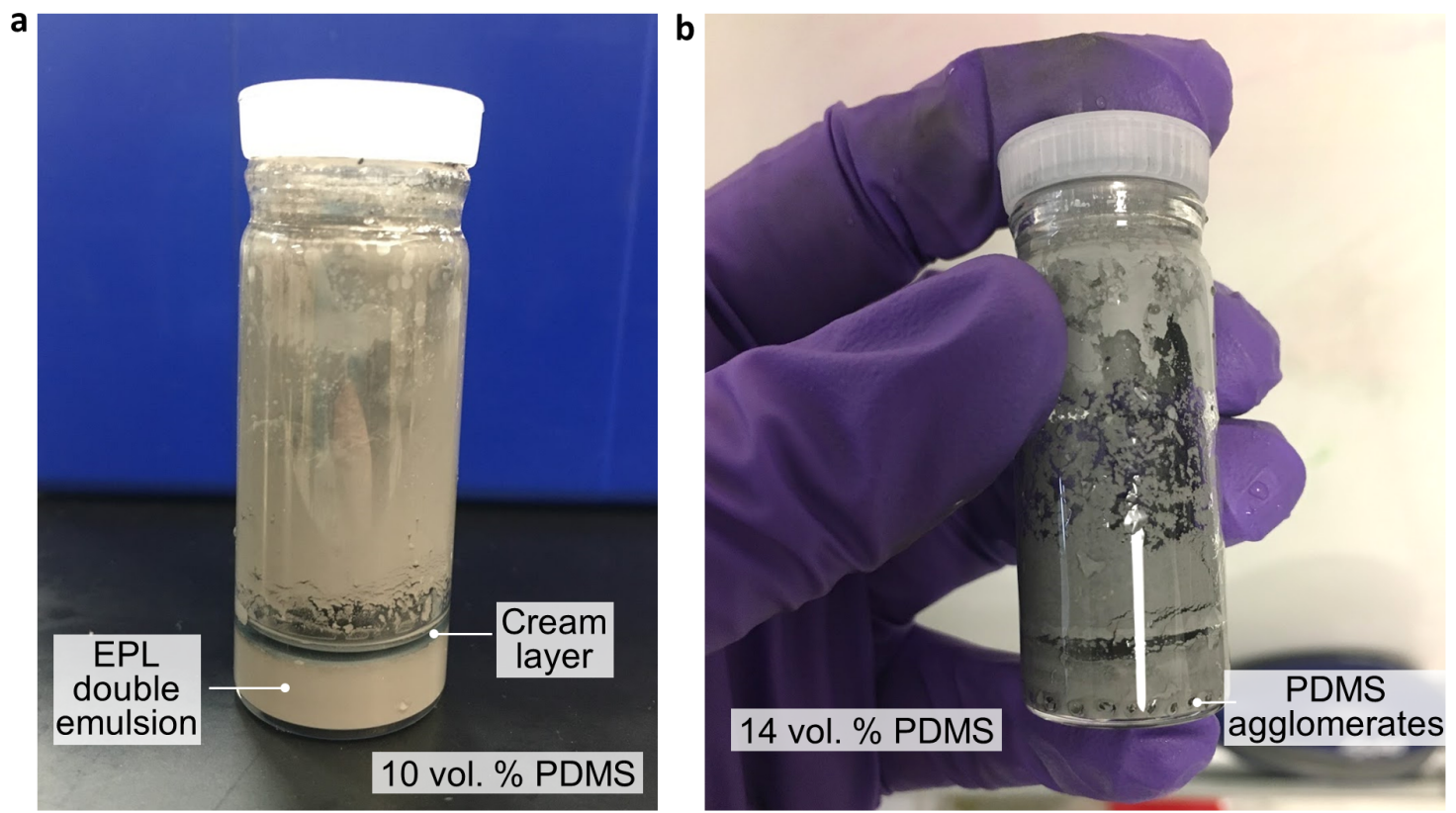

Figure S1. Optical images of EtOH/PDMS/LM double emulsion with (a) 10 vol \% and (b) 14 vol \% of PDMS precursors. a, The cream layer is consisted of residual PDMS precursors and a suspension of lighter liquid metal particles in ethanol. The EPL double emulsion is collected after removing the cream layer. $\mathbf{b}$, The excessive amount of PDMS precursors results in agglomerates during sonication.
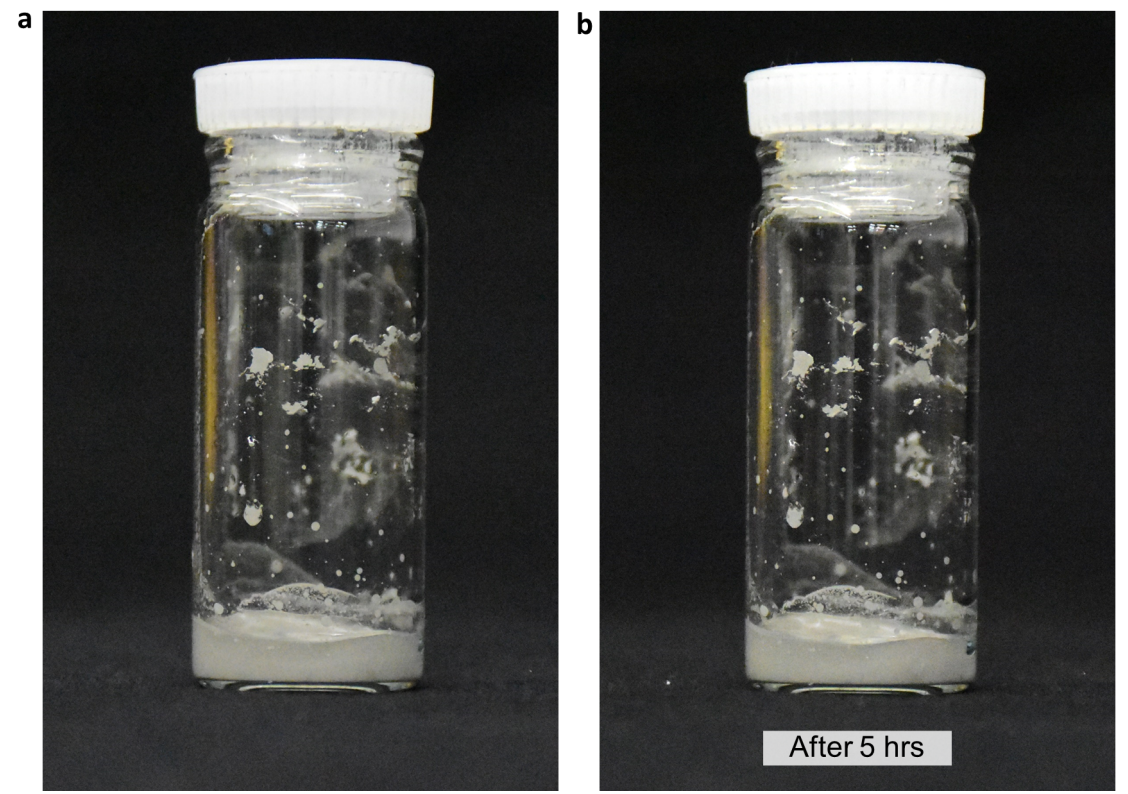

Figure S2. Optical images of EPL double emulsion with 10 vol \% of PDMS precursors before (a) and after 5 hrs (b). No agglomeration or sedimentation appeared to occur. 

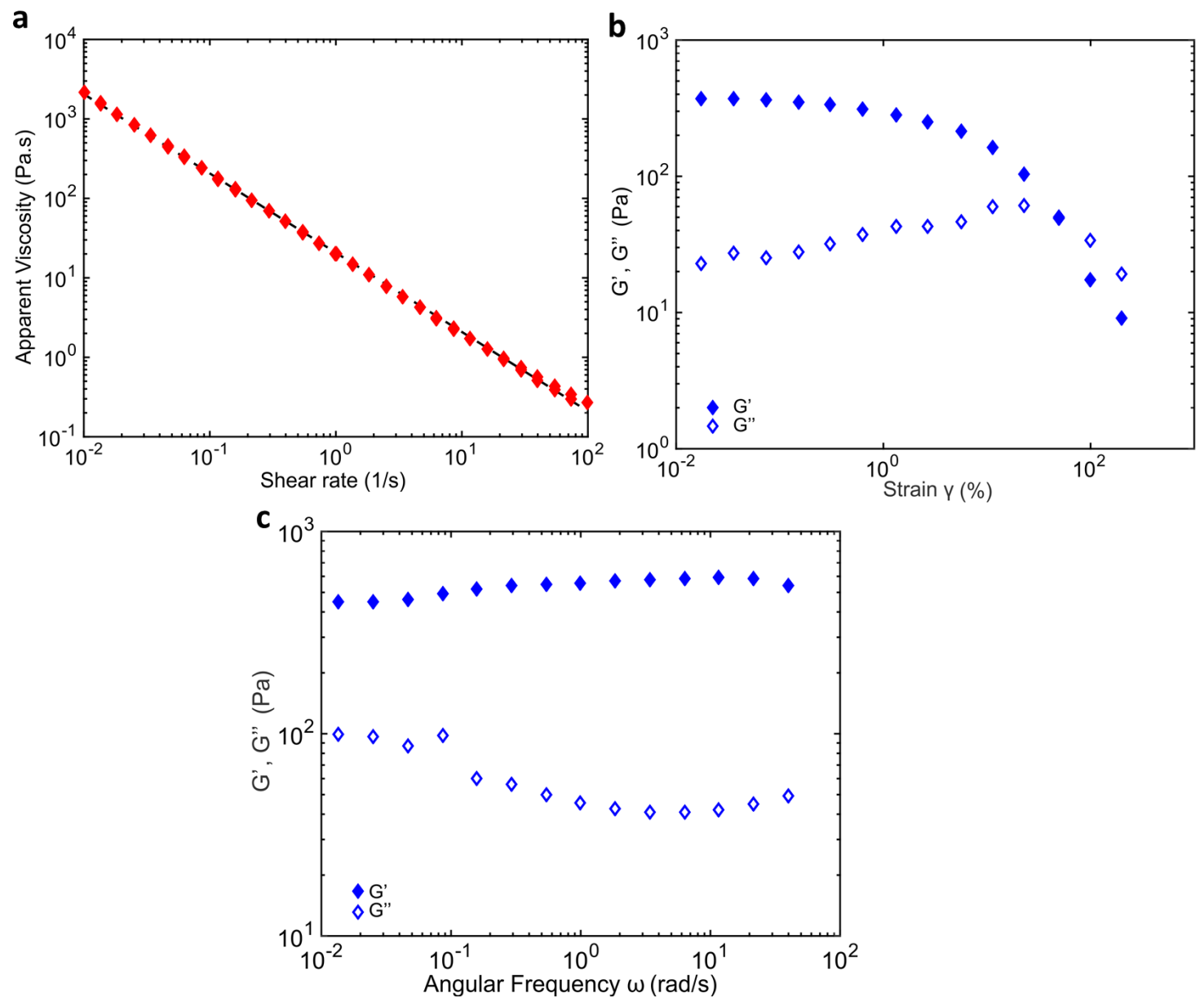

Figure S3. Rheological properties of EPL double emulsion with 10 vol\% PDMS. a, Apparent viscosity as a function of shear rate. The material exhibits a pronounced shear thinning behavior with an apparent viscosity of $2146 \mathrm{~Pa} \cdot s$ at a shear rate of $0.01 \mathrm{~s}^{-1}$. b. Storage modulus (G') and loss modulus (G") as a function of strain sweep at a constant frequency of $1 \mathrm{rad} / \mathrm{s}$. The result indicates that the ink is transformed from a dominantly solid-like to a liquid-like state when the strain is increased beyond $49 \%$. c, Storage modulus (G') and loss modulus (G") as a function of angular frequency at a constant strain amplitude of $0.5 \%$. The ink exhibits a solid-like response manifested by the dominance of the storage modulus G' over the loss modulus G" in the entire frequency range.

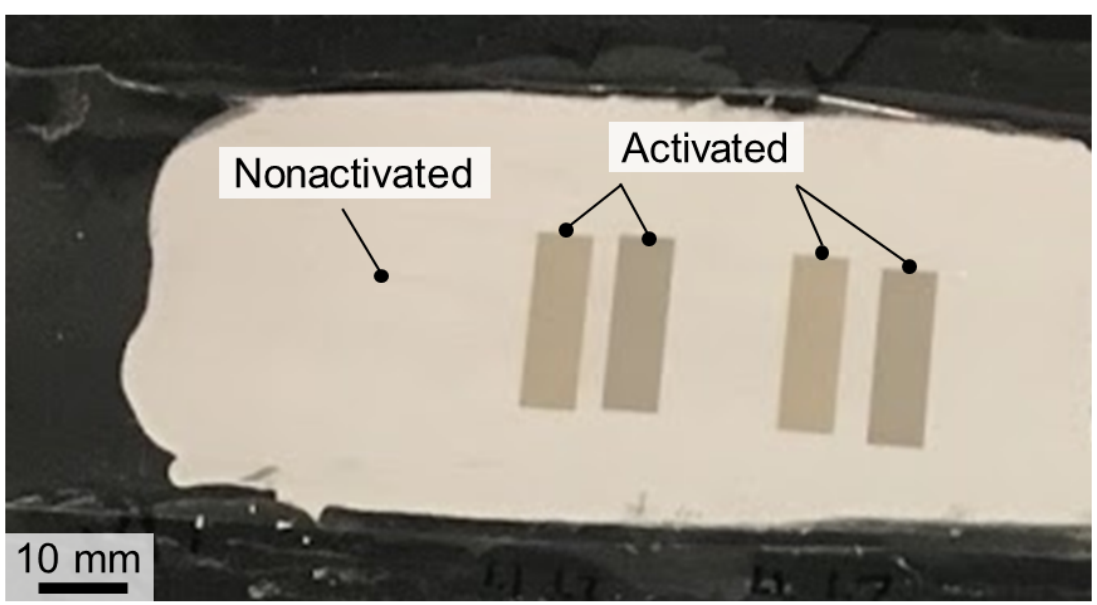

Figure S4. Optical image of a PDMS-LM composite film. The rectangles in dark color indicate laser-activated regions where the pristine, non-conductive structure is coalesced into a continuously conductive, complex network. 

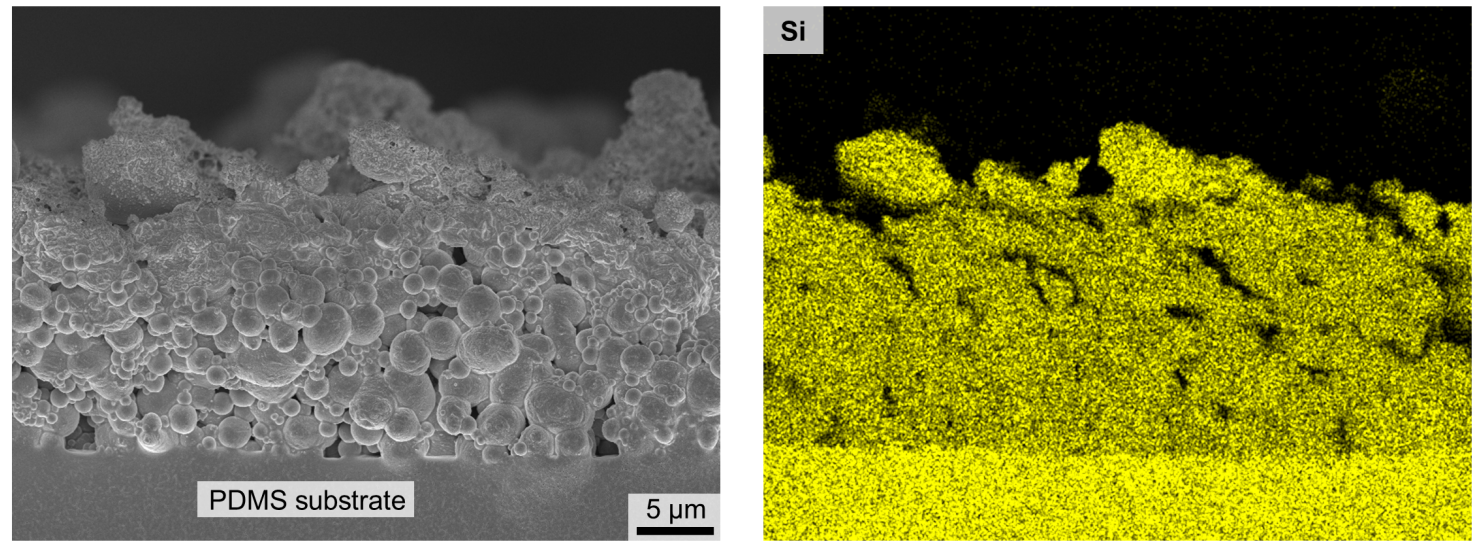

Figure S5. EDS Si mapping of the cross-sectional surface of a laser-activated PDMS-LM composite. The result shows that $\mathrm{Si}$ element is distributed across the entire surface, which supports that the cured PDMS precursors and the PDMS substrate is continuously bonded. The PDMS acts as an adhesive, linking the top to the bottom.
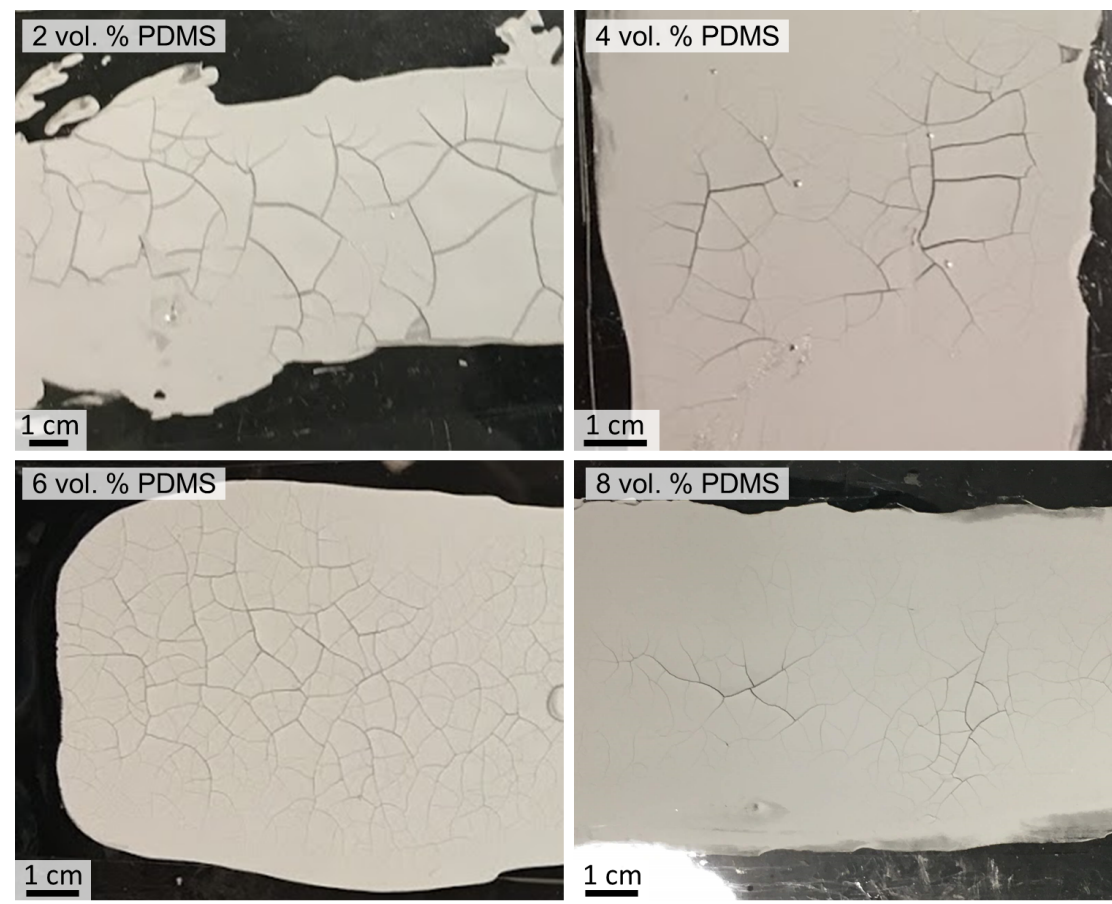

Figure S6. Optical images of an as-printed EPL double emulsion with 2 vol \%, 4 vol \%, 6 vol \%, and 8 vol \% PDMS precursors on PDMS substrates. The composite films exhibit cracks resulting from particle aggregation during EtOH evaporation. 


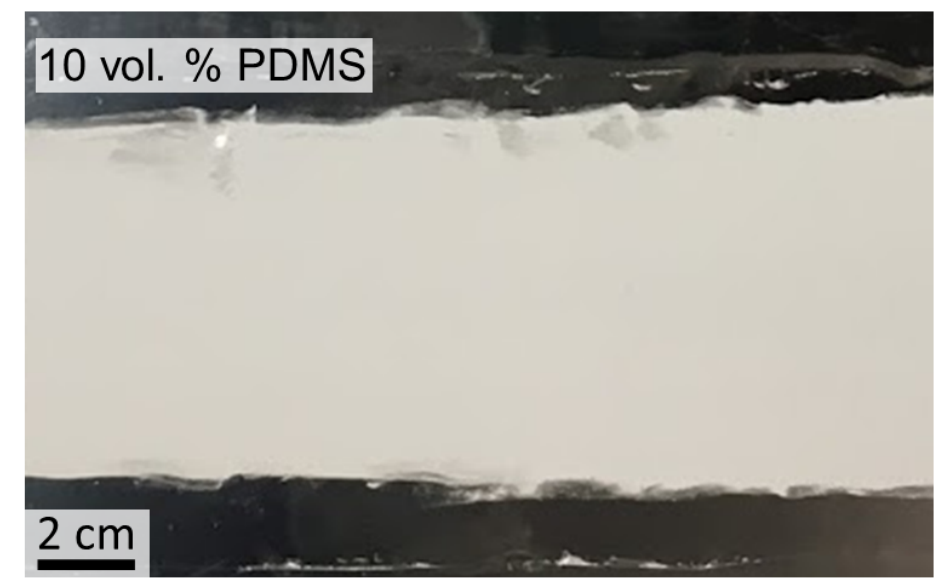

Figure S7. Optical image of an as-printed EPL double emulsion with 10 vol \% PDMS precursors on a PDMS substrate. The as-printed composite film is thin, uniform, and crack-free.

\section{Analytical estimation of capillary force}

When printing the EPL double emlulsion onto a silicone substrate, the EtOH evaporates rapidly, releasing the PDMS-LM droplets onto the substrate. The LM microparticles relax, and the encapsulating PDMS precursors flow, forming meniscus shaped liquid PDMS bridges between the particles. The schematic of the capillary interaction between two liquid metal particles connected with a PDMS liquid bridge is shown in Figure S5a. The capillary force $F$ between two spheres can be estimated analytically by: ${ }^{1}$

$$
F=-\frac{2 \pi R \gamma \cos \theta}{1+H / 2 d}-2 \pi \gamma R \sin \alpha \sin (\theta+\alpha),
$$

where $R$ is the radius of the sphere, $\gamma$ is the surface tension of the liquid, $\theta$ is the contact angle, $\alpha$ is the embracing angle, $H$ is separation distance between the two spheres, and $d$ is the immersion length given by:

$$
d=(H / 2) \times\left[-1+\sqrt{1+2 V /\left(\pi R H^{2}\right)}\right],
$$

where $V$ is the volume of the liquid bridge between the two spheres, which is given by:

$$
V=\pi R^{2} \alpha^{2} H+0.5 \pi R^{3} \alpha^{4}
$$

We assume the radius of the spheres of equal size, which are the measured average particles size $R=0.955 \mu \mathrm{m}$ (obtained from the particle analysis results in Figure 2c), and the surface tension of PDMS is $20.4 \mathrm{mN} / \mathrm{m}^{2}$ The contact angle $\theta$ and the embracing angle $\alpha$ are estimated to be $30^{\circ}$ and $40^{\circ}$ respectively by measuring from the EDS Si mapping in Figure 2d.

The gravitational force $G$ of a liquid bridge between two spheres is given by:

$$
G=\rho V g
$$



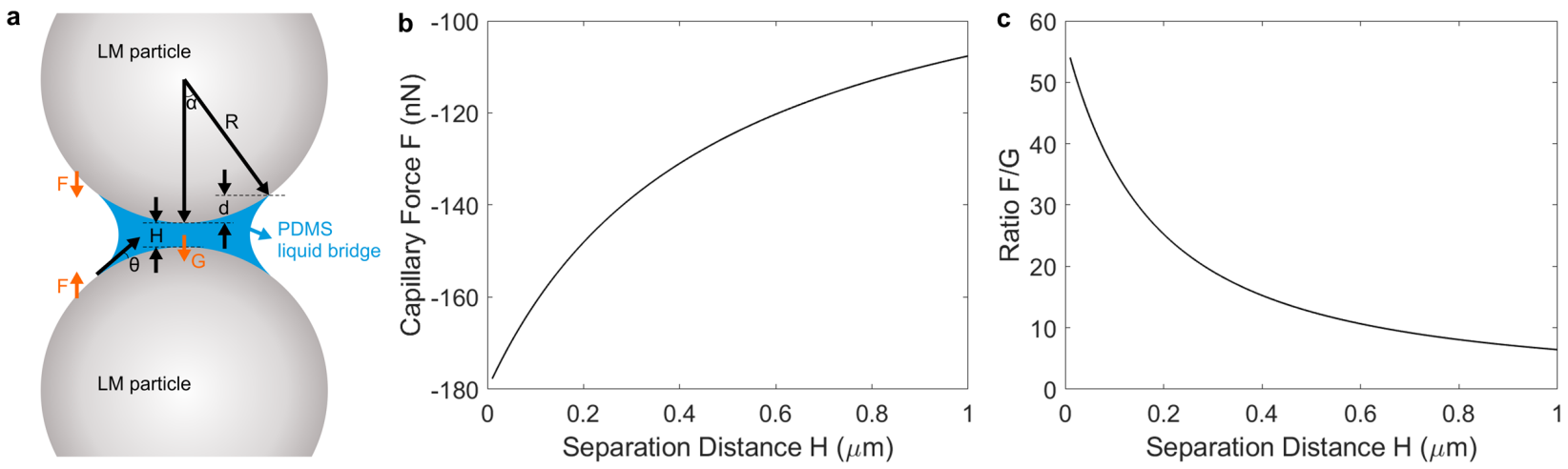

Figure S8. Analytical estimation of capillary force. a, Schematic of the capillary interaction between two liquid metal particles connected with a PDMS liquid bridge. b, Capillary force, and the ratio of capillary force to gravitational force as a function of separation distance between two liquid metal particles.

where $\rho$ is the density of PDMS, which is $0.99 \mathrm{~g} / \mathrm{cm}^{3}{ }^{3}$

From equation (1) and equation (4), the ratio of capillary force to gravitational force is give by:

$$
\begin{aligned}
R a & =\frac{F}{G} \\
& =\frac{-\frac{2 \pi R \gamma \cos \theta}{1+H / 2 d}-2 \pi \gamma R \sin \alpha \sin (\theta+\alpha)}{\rho V g},
\end{aligned}
$$

Taking separating distances $H$ ranging from $0.01 \mu \mathrm{m}$ to $1 \mu \mathrm{m}$, the volume of the liquid bridge $V$ and the immersion length $d$ can be estimated using equation (3) and equation (2). Plugging equation (2) into equation (1) and equation (5) yields the estimation of the capillary force, and the ratio of capillary force to gravitational force as a function of separation distance.

Figure S5b shows that as two particles get closer to each other (separation distance $H$ decreases), the capillary force increases. In addition, Figure S5c shows that the capillary force between two particles is significantly larger than the gravitational force, which indicates that the PDMS precursor can hold the particles together rather than completely dripping from the top to the bottom substrate. Therefore, when the PDMS precursors cure, they act as an adhesive, bonding the particles to each other and to the surfaces of the PDMS substrates. 

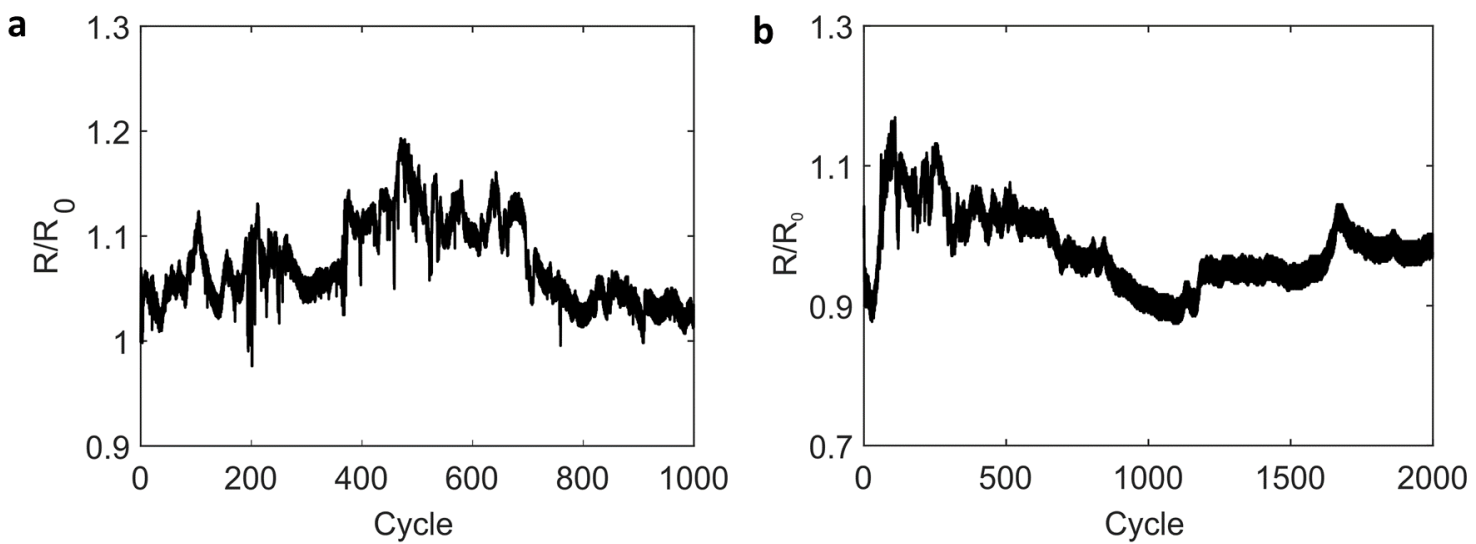

Figure S9. Relative resistance change of the laser-activated PDMS-LM composites with 4 vol \% (a), and 8 vol \% (b) PDMS content (less than the optimal value 10 vol \%), subjected to uniaxial tensile cyclic loading to $100 \%$. The composites exhibited inconsistent and unstable electrical responses.

\begin{tabular}{|c|c|c|c|}
\hline LM-based conductors & Conductivity $\left(\mathrm{S} \mathrm{m}^{-1}\right)$ & $\mathrm{R} / \mathrm{R}_{0}$ at $\mathrm{x} \%$ strain & Fabrication process \\
\hline Bulk eGaIn (theoretical) & $3.4 \times 10^{6}$ & 4 at $100 \%$ strain & - \\
\hline PDMS-LM composite (this work) & $7.7 \times 10^{5}$ & 1.2 at $100 \%$ strain & Emulsion processing, Laser sintering \\
\hline PDMS-galinstan $^{4}$ & $1.05 \times 10^{4}$ & 1.27 at $100 \%$ strain & Shear mixing, Mechanical sintering \\
\hline AgNP-Ga-In ${ }^{5}$ & $4.8 \times 10^{6}$ & 2.3 at $86 \%$ strain & Inkjet printing, Rubbing, Cleaning \\
\hline LM-silicone $^{6}$ & - & 2.3 at $100 \%$ strain & Shear mixing, Centrifuging \\
\hline
\end{tabular}

Supplementary Table S1. Electrical conductivity, relative resistance change over strain, and fabrication process of PDMS-LM composite and other LM-based conductors in the literature. The PDMS-LM composite exhibits a superior balance of strain-insensitivity and electrical conductivity.

\section{References}

1. Yakov I Rabinovich, Madhavan S Esayanur, and Brij M Moudgil. Capillary Forces Between Two Spheres with a Fixed Volume Liquid Bridge: Theory and Experiment. Langmuir, 21(24):10992-10997, 2005.

2. Souheng Wu. Interfacial and Surface Tensions of Polymers. Journal of Macromolecular Science-Reviews in Macromolecular Chemistry, 10(1):1-73, 1974.

3. SEMICOSILR 964.

4. A. Fassler and C. Majidi. Liquid-Phase Metal Inclusions for a Conductive Polymer Composite. Adv. Mater., 27:1928-1932, 2015.

5. Mahmoud Tavakoli, Mohammad H Malakooti, Hugo Paisana, Yunsik Ohm, Daniel Green Marques, Pedro Alhais Lopes, Ana P Piedade, Anibal T de Almeida, and Carmel Majidi. EGaIn-Assisted Room-Temperature Sintering of Silver Nanoparticles for Stretchable, Inkjet-Printed, Thin-Film Electronics. Advanced Materials, 30(29):1801852, 2018.

6. Lu-yu Zhou, Jian-zhong Fu, Qing Gao, Peng Zhao, and Yong He. All-Printed Flexible and Stretchable Electronics with Pressing or Freezing Activatable LiquidMetal-Silicone Inks. Advanced Functional Materials, 30(3):1906683, 2020. 